\title{
The link between resting heart rate variability and affective flexibility
}

\author{
Maud Grol ${ }^{1} \cdot$ Rudi De Raedt ${ }^{1}$ \\ Published online: 27 May 2020 \\ (C) The Psychonomic Society, Inc. 2020
}

\begin{abstract}
The neurovisceral integration model aims to account for the complex interplay between physiological, cognitive, and emotion regulation processes through their support by common cortico-subcortical neural circuits. According to the model, vagally mediated heart rate variability (HRV) serves as a peripheral index of the functioning of these circuits, with higher levels of resting HRV reflecting more optimal functioning, to support goal-directed behaviour and adaptability to environmental demands. Although increased cognitive flexibility has been related to higher resting HRV, this has not been assessed in the context of emotional information to examine the interplay between cognition and emotion. Therefore, we investigated $(n=109)$ the relationship between resting HRV and performance on a task-switching paradigm in which participants shift attention between affective and nonaffective aspects of emotional material. Resting HRV was not associated with flexibility in processing of positive material, but more efficient shifting of attention (greater flexibility) from affective to nonaffective aspects of negative information was related to lower resting HRV. The avoidance theory of worry and anxiety, as well as empirical evidence, links anxiety to attentional avoidance of negative information. Our findings therefore support the neurovisceral integration model such that when greater flexibility can facilitate attentional avoidance of negative information — as seen in anxiety — it is related to lower resting HRV.
\end{abstract}

Keywords Heart rate variability $\cdot$ RMSSD $\cdot$ Vagal tone $\cdot$ Cognitive flexibility $\cdot$ Affective flexibility

The neurovisceral integration model proposes that physiological, emotion, and cognitive regulation processes are related to each other in the service of goal-directed behaviour and adaptability to changing environmental demands (Thayer, Hansen, Saus-Rose, \& Johnsen, 2009; Thayer \& Lane, 2000). The interplay between these factors can contribute to individual differences in (mental) health and disease. The model summarizes the relationship between the central nervous system (CNS) and the autonomous nervous system (ANS), and puts forward a common cortico-subcortical neural circuit that serves as the structural link between these regulation processes. A network of neural structures (for overviews, see Thayer, Åhs, Fredrikson, Sollers, \& Wager, 2012; Thayer et al., 2009; Thayer \& Lane, 2000) consisting, amongst others, of

Electronic supplementary material The online version of this article (https://doi.org/10.3758/s13415-020-00800-w) contains supplementary material, which is available to authorized users.

Maud Grol

maud.grol@ugent.be

1 Department of Experimental Clinical and Health Psychology, Ghent University, Ghent, Belgium prefrontal areas, including ventromedial prefrontal cortex and anterior cingulate cortex, and subcortical areas such as the hypothalamus and amygdala, are together called the central autonomic network. This central autonomic network regulates the ANS through sympathetic and parasympathetic (vagal nerve) branches that innervate the heart (Appelhans \& Luecken, 2006; Benarroch, 1993). It is the interaction between the sympathetic and parasympathetic subsystems of the ANS that has a prominent influence on cardiac activity and regulates the time between consecutive heartbeats. This dynamic balance between the sympathetic and parasympathetic branch allows for flexible control over the response of the body (e.g., heart) to a range of external and internal stimuli. An increase in heart rate could result from activation of sympathetic fibers or decreased parasympathetic inhibition (vagal withdrawal). The parasympathetic system is more dominant in maintaining resting heart rate. Whereas sympathetic influence on heart rate unfolds in a relatively slower manner, parasympathetic regulation of the heart is much faster, allowing for momentary modulation of cardiac activity (Pumprla, Howorka, Groves, Chester, \& Nolan, 2002). Heart rate variability (HRV) is the variation in time intervals between heart beats and provides an index of this parasympathetic influence on the heart (Laborde, Mosley, \& Thayer, 2017). As the vagus nerve is the primary 
parasympathetic nerve (Brodal, 2010), when we refer to HRV, we always refer to vagally mediated HRV.

The central autonomic network regulates this autonomic influence on heart rate (Benarroch, 1993), and its output is thus directly linked to HRV (Thayer et al., 2009; Thayer \& Lane, 2000). Thayer et al. (2012; Thayer et al., 2009; Thayer et al., 2000) have reported considerable structural overlap of the central autonomic network and neural circuits supporting cognitive and emotion regulation processes that allow for goal-directed behaviour and flexible adaptation to changing environmental demands. The neurovisceral integration model postulates that HRV serves as a peripheral index of the functional capacity of these cortico-subcortical neural circuits and higher levels of resting HRV (measured when individuals are doing nothing) are believed to reflect more optimal functioning of this network (Thayer et al., 2012). In support of this idea they show that cognitive, emotion, and physiological regulation are associated with cardiac vagal tone as measured by resting HRV (Thayer et al., 2009; Thayer \& Lane, 2000).

The same neural circuits that have been linked to HRV (Thayer et al., 2012; Thayer et al., 2009; Thayer \& Lane, 2000) are indeed also underlying regulation of emotion, and dysfunctions in parts of this circuitry have been associated with psychopathology (Davidson, Pizzagalli, Nitschke, \& Putnam, 2002). Previous work has shown a relationship between higher resting HRV and better emotion regulation (Appelhans \& Luecken, 2006; Balzarotti, Biassoni, Colombo, \& Ciceri, 2017; Ruiz-Padial, Sollers, Vila, \& Thayer, 2003; Thayer \& Brosschot, 2005). On the other hand, disorders of emotion dysregulation, such as anxiety, are proposed to be characterized by a rigid coupling of the CNSANS system, reflected by elevated sympathetic activity and reduced parasympathetic (vagal) control as measured by vagally mediated HRV (for review, see Friedman, 2007). Anxiety indeed seems to be characterized by the inability to inhibit responses to threat that have, amongst others, cognitive, behavioural (e.g., avoidance), affective (e.g., fear), and autonomic (e.g., HR increase) components (Friedman, 2007). Several studies find that anxiety, both in nonpathological and pathological form, is associated with reduced resting HRV levels (Friedman, 2007). Worry, which is a form of perseverative thinking and a core cognitive feature of generalized anxiety disorder (GAD), has also shown to lower phasic HRV levels in both GAD patients and healthy controls (Thayer, Friedman, \& Borkovec, 1996). Similarly, it has been shown that lower levels of resting HRV are correlated with maladaptive forms of perseverative thinking (i.e., brooding and depressive rumination), and it has been demonstrated that this thinking style partially mediates the relationship between lower resting HRV and anxiety (Williams et al., 2017).

The neurovisceral integration model aims to account for the complex interaction between physiological, affective, and cognitive processes. Besides the link between HRV and emotion (dys-)regulation, research has therefore also focused on the role of individual differences in resting HRV and executive function. Executive functions are a set of cognitive control processes consisting of inhibition of prepotent responses, mental set shifting (i.e., cognitive flexibility), and updating and monitoring of information in working memory (Miyake et al., 2000). These processes allow flexible adaptation of behaviour and cognition in line with one's goals and changing environmental demands. Cognitive flexibility has therefore been proposed as one of the cognitive mechanisms underlying effective emotion regulation (Genet \& Siemer, 2011; Ochsner \& Gross, 2007). On the other hand, deficits in cognitive flexibility have been associated with trait anxiety and GAD, which are characterized by emotion dysregulation (Ansari, Derakshan, \& Richards, 2008; Kim et al., 2019; Lee \& Orsillo, 2014), although this has not always been replicated (Han et al., 2016). Executive functions rely on a prefrontalparietal network (Niendam et al., 2012) that partially overlaps with the neural circuits associated with HRV and emotion processing (Thayer et al., 2012), and this common corticosubcortical neural network may thus serve as the link between cognitive, emotional, and physiological processes.

Previous research has linked higher levels of resting HRV to better performance on tasks of monitoring and updating working memory, attention control, and response inhibition (Thayer et al., 2009, for a review; Zahn et al., 2016, for a meta-analysis). Although most research has investigated the link between HRV and executive functions of inhibition and updating and monitoring working memory, few studies have measured cognitive flexibility (i.e., mental set shifting). In a study with a sample of patients with panic disorder, it has been shown that higher resting HRV is associated with better performance on the Wisconson Card Sorting Test, a measure of set shifting that also requires working memory (Hovland et al., 2012). A more recent study in an unselected sample used a task-switching paradigm in which cognitive flexibility is reflected by switch costs - an increase in reaction time (RT) on task-switching trials as compared with task-repetition trials. The authors observed a relationship between higher levels of resting HRV and smaller switch costs (i.e., greater cognitive flexibility; Colzato, Jongkees, de Wit, van der Molen, \& Steenbergen, 2018).

The links across vagally mediated HRV, cognitive flexibility and emotion (dys)regulation fit well with the neurovisceral integration model (Thayer et al., 2009; Thayer \& Lane, 2000). However, previous research has typically measured cognitive flexibility with tasks using nonaffective stimuli, but it may be particularly interesting to assess cognitive flexibility in the context of affective information and how this is related to HRV - especially as disorders of emotion dysregulation, such as depression and anxiety, are associated with a biased processing of affective information (for reviews see Cisler \& Koster, 2010; Joormann \& Stanton, 2016). This allows to 
really examine the interplay between physiological regulation, emotion, and cognitive control.

Cognitive flexibility in the context of affective information is here referred to as affective flexibility, and has been measured with a task-switching paradigm where individuals have to sort positive and negative images according to an affective rule (i.e., valence of the picture) or a nonaffective rule (i.e., the number of people depicted; Genet, Malooly, \& Siemer, 2013; Malooly, Genet, \& Siemer, 2013). More flexibility (i.e., lower switch costs) when shifting attention towards nonaffective aspects of negative information and towards affective aspects of positive information has been shown to predict more effective reappraisal when instructed to use this emotion regulation strategy (Malooly et al., 2013). In another study, a relationship was found between less flexibility when shifting attention towards nonaffective aspects of negative information and rumination (Genet et al., 2013). Interestingly, less flexibility when shifting attention towards nonaffective aspects of positive information was associated with lower rumination (Genet et al., 2013), highlighting that more flexibility is not adaptive per se and may depend on the context.

Recent unpublished data has also shown that when assessing cognitive flexibility in the context of affective information, more flexibility is not necessarily more adaptive. Whilst more flexibility when shifting attention from nonaffective towards affective aspects of positive information was associated with reductions in anxiety across 7 weeks, more flexibility when shifting attention towards nonaffective aspects of negative information was associated with greater increases in anxiety and worry over time (Twivy, Grol, \& Fox, 2019). Although this latter finding seems to contradict previous work on attention facilitation to negative information in anxiety, it is actually consistent with findings of a relationship between anxiety and attentional avoidance of negative stimuli at relatively longer presentation times (Cisler \& Koster, 2010). Such attentional avoidance is believed to reflect a strategic process underlying emotion regulation goals (Cisler \& Koster, 2010), but it actually leads to the maintenance of anxiety. The model of avoidance in anxiety and worry (Borkovec, Alcaine, \& Behar, 2004) proposes that worry functions as a cognitive avoidance response to (perceived) future threats. It functions as a cognitive attempt to generate ways to anticipate or prevent bad things from happening, and by shifting attention to worrisome thinking when threatening information is detected, a suppression of somatic arousal occurs. The cognitive avoidance response is therefore negatively reinforced, but it precludes emotion processing of the fear-related information which is necessary for extinction of anxiety responses, leading to a maintenance of worry and anxiety (Borkovec et al., 2004). In support of this, a recent study in children (Lester, Lisk, Carr, Patrick, \& Eley, 2019) comparing 'toward threat' with 'avoidance of threat' attention training found that a larger change in attention bias toward threat (but not away from threat) predicted a greater reduction in anxiety across time. The authors argued that attention bias to threat can facilitate learning that negative information does not always result in an aversive outcome (in line with exposure therapy).

It thus seems that affective information can create a 'context' in which more cognitive flexibility, in terms of mental set shifting, is not necessarily more adaptive. In keeping with the neurovisceral integration model's aim to account for the complex interaction between physiological, affective, and cognitive processes (Thayer et al., 2009; Thayer \& Lane, 2000), we therefore aimed to investigate the association between resting HRV and cognitive flexibility in the context of affective information. We measured affective flexibility with a taskswitching paradigm (Malooly et al., 2013) and used the time-domain-based root mean square successive difference (RMSSD) in beat-to-beat intervals as our measure of resting state HRV. The RMSSD and the frequency-domain-based measure of high-frequency changes in heart beat (HF-HRV) are often highly correlated and both are reliable measures of vagally mediated HRV, but the RMSSD is relatively free of respiratory influences as compared with HF-HRV (Laborde et al., 2017).

Previous research has associated more efficient shifting (i.e., greater flexibility) from nonaffective aspects towards affective aspects of positive information with greater reappraisal efficacy (Malooly et al., 2013) and reductions in anxiety over time (Twivy et al., 2019). Research on resting HRV has associated anxiety with reduced resting HRV (Friedman, 2007), whereas individuals with greater emotion regulation ability have shown higher levels of resting HRV (Appelhans \& Luecken, 2006; Thayer \& Brosschot, 2005). Bringing these previous findings together, we expected greater flexibility (i.e., lower RT switch costs) when shifting attention from nonaffective towards affective aspects of positive information to be associated with higher resting HRV. In contrast, more efficient shifting of attention from affective to nonaffective aspects of negative information, possibly facilitating avoidance of affective aspects of negative information, has been associated with greater increases in anxiety over time (Twivy et al., 2019). Based on these findings and models of attentional avoidance of threat in anxiety and worry (Borkovec et al., 2004; Cisler \& Koster, 2010), we therefore expected more efficient (i.e., lower RT switch costs) shifting of attention from affective to nonaffective aspects of negative information to be associated with lower resting HRV. Previous findings with the affective flexibility task and recent findings associating resting HRV with increased cognitive flexibility (Colzato et al., 2018) are all based on switch costs in RT, and no effects were found with switch costs in accuracy. Our hypotheses are therefore focused on switch costs in RT. Given that HRV has been shown to be modulated by age (Umetani, Singer, McCraty, \& 
Atkinson, 1998), gender (Sztajzel, Jung, \& Bayes de Luna, 2008; Umetani et al., 1998), anxiety (Friedman, 2007), depression (Kemp et al., 2010), stress (Dishman et al., 2000), and heart rate (Gąsior, Sacha, Jeleń, Zieliński, \& Przybylski, 2016; Monfredi et al., 2014), we examined the relationship of these variables with HRV in the current study. We controlled for those variables that significantly correlated with resting HRV.

\section{Method}

\section{Participants}

A total of 120 participants were recruited by convenience sampling. ${ }^{1}$ Beat-to-beat heart rate recordings of 10 participants were very noisy, and this data was therefore excluded from further analyses. Additionally, one participant was excluded from analysis because the percentage of errors in the switching task was more than three standard deviations from the sample mean. The final sample therefore consisted of 83 females and 26 males between the ages of 18 and 53 years ( $M$ $=23.19, S D=6.26)$. Exclusion criteria included current or a history of cardiovascular disease, seeking help or being in treatment for psychological/psychiatric complaints in the past 6 months, and use of psychoactive medication or medication that influences cardiovascular activity. The majority of participants in the sample were university students. Participants were paid for their participation. This study was approved by the Medical Ethics Committee at the Ghent University Hospital (reference: EC/2018/1505).

\section{Material}

Questionnaires Trait anxiety was measured using the trait component of the State-Trait Anxiety Inventory (STAI-T; Spielberger, Gorsuch, Lushene, Vagg, \& Jacobs, 1983; Van der Ploeg, Defares, \& Spielberger, 2000). Participants are asked to rate how they generally feel on a 4-point scale from 1 (almost never) to 4 (almost always). A total score is obtained between 20 and 80 . In the present study, the STAI-T had high internal consistency $(\alpha=0.91)$.

The Beck Depression Inventory (BDI; Beck, Steer, \& Brown, 1996; Van der Does, 2002) was used to measure presence and severity of depressive symptoms during the past 2 weeks. Participants rate items on a $0-3$ scale. A total score is obtained between 0 and 63 . In the present study, the BDI had high internal consistency $(\alpha=0.91)$.

\footnotetext{
${ }^{1}$ This data are part of a larger study on the link between affective flexibility and stress reactivity for which an a-priori power analysis was done. For the current analysis, asssuming a medium effect size, alpha level of 0.05 , and power of 0.80 , the necessary sample size based on the $R^{2}$ increase when adding switch costs to the model is 85 participants.
}

We measured mood state with visual analogue scales $(0-10$ $\mathrm{cm}$, resulting in a 0-100 scale) measuring how happy, sad, aroused, angry, and tense participants were feeling "at this moment." We measured how happy and sad participants were feeling on a scale from neutral to as happy/sad as I can imagine, and we measured arousal on a scale from calm to aroused. Anger and tenseness were measured on a scale from not at all to as angry/tense as I can imagine.

Affective flexibility Affective flexibility was measured using a task-switching paradigm based on Malooly et al. (2013). In this task, participants have to sort emotional images according to an affective task rule or a nonaffective task rule. For the affective task rule participants are instructed to sort the images according to whether the depicted scene is positive or negative. For the nonaffective task rule, participants have to indicate whether one or no person $(\leq 1)$ is depicted versus two or more $(\geq 2)$ depicted people. Even if only part of a person is depicted, this counts as well. Images were selected from the International Affective Picture System (IAPS; Lang, Bradley, \& Cuthbert, 2008). Forty images were selected for each of the following categories: positive with one or fewer people, positive with two or more people, negative with one or fewer people, and negative with two or more people (i.e., $160 \mathrm{im}$ ages in total). Positive and negative images differed in valence ratings, but were balanced in terms of arousal ratings. An additional 20 images were used in the practice blocks.

Each trial started with a black blank screen $(250 \mathrm{~ms})$, followed by the presentation of a central fixation cross (250 ms). See Fig. 1 for an example trial sequence. Next an emotional image was presented in the centre, and cues indicating the relevant task rule appeared on either side of the image: ' + ' and '-' for the affective task rule and ' $\leq 1$ ' or ' $\geq 2$ ' for the nonaffective task rule. This was presented until the participant responded or for $5000 \mathrm{~ms}$, whichever was sooner. The background colour (white or grey) of the screen during presentation of the emotional image and cues also indicated the sorting rule. Participants had to respond by pressing one of two adjacent keys on the keyboard ( $N$ and $M$ labelled as ' $L$ ' and ' $\mathrm{R}$ '). Instructions told participants to work quickly, but try to be as accurate as possible.

The task started with two practice blocks, each consisting of 10 trials, in which participants had to first apply the affective rule only, followed by the second block using the nonaffective rule only. The test phase consisted of two 160-trial blocks with a break in between. Trials were presented in a pseudorandom order (Malooly et al., 2013). There were eight versions of the task, counterbalancing different combinations of cue to key mappings and rule (affective/nonaffective) to background colour (grey/white) mappings. 


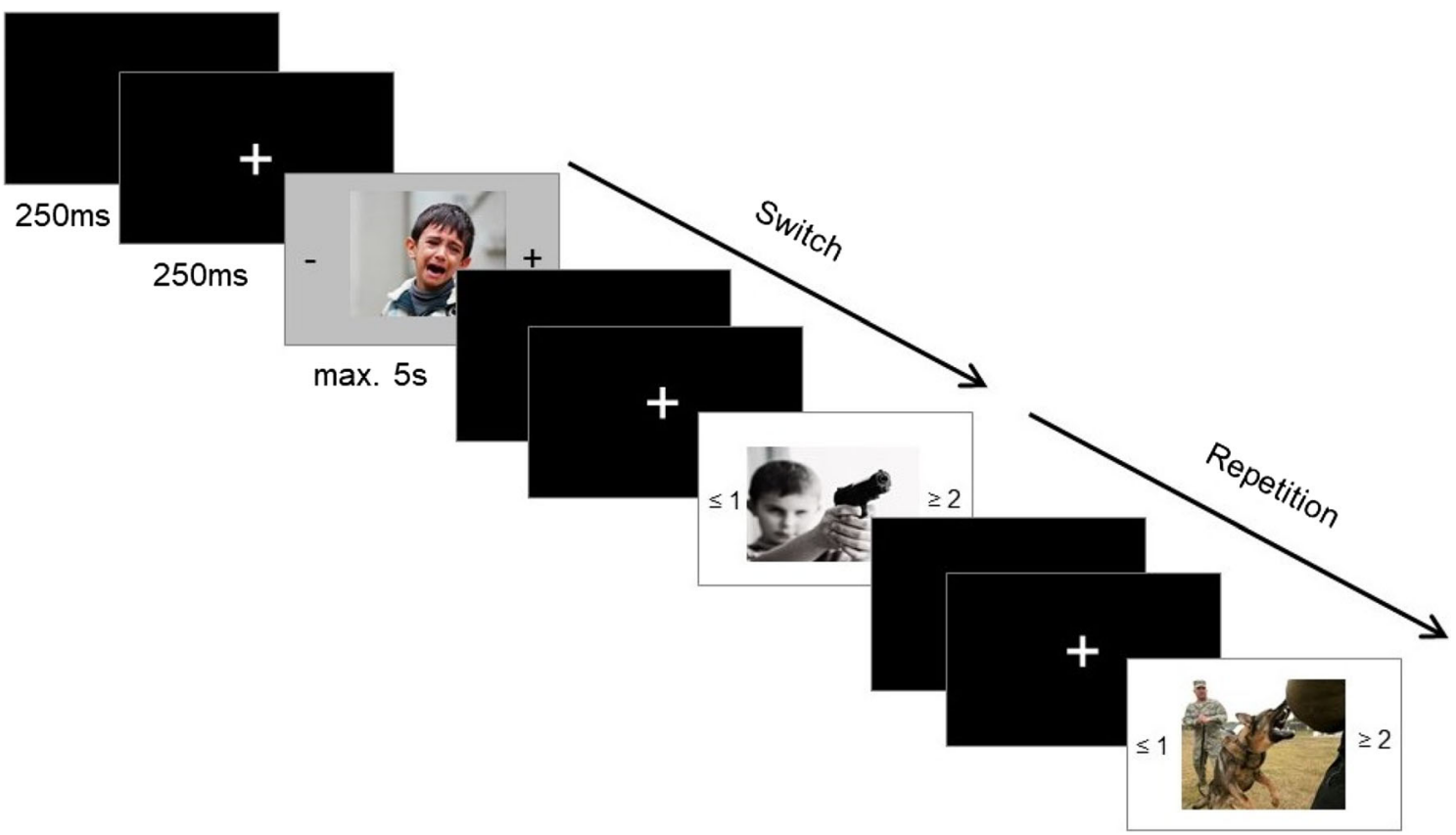

Fig. 1 Example of a trial sequence from the task-switching paradigm, with a nonaffective negative switch trial. The images displayed are for illustrative purposes and are not part of the International Affective Picture System

Calculation of switch costs ${ }^{2}$ The difference between mean RT on switch trials and repetition trials is termed the switch cost. Four different types of switch costs were calculated following Malooly et al. (2013): nonaffective negative switch costs, affective negative switch costs, nonaffective positive switch costs, and affective positive switch costs. Mean RT on repetition trials were subtracted from mean RT on switch trials. For example, affective positive switch costs were calculated by subtracting the mean RT on trials involving a repetition of the affective task rule in the presence of positive images from the mean RT on trials involving a switch away from processing the nonaffective aspects towards the affective aspects in the presence of positive images. Negative nonaffective switch costs were calculated by subtracting the mean RT on trials involving a repetition of the nonaffective task rule in the

\footnotetext{
${ }^{2}$ Previous studies have used slightly different ways to calculate the switch costs. Genet et al. (2013) calculated nonaffective switch costs by subtracting RTs on trials in which the affective rule was repeated from RTs on trials in which the task switched from the affective rule to the nonaffective rule. Affective switch costs were calculated in an analogous manner. On the other hand, in the paper of Malooly et al. (2013), nonaffective switch costs were calculated by subtracting RTs on trials in which the nonaffective rule was repeated from RTs on trials in which the task switched from the affective rule to the nonaffective rule. We use the same method as Malooly et al. (2013) to compare performance on trials where the same task rule has to be performed and which are either preceded by the same task rule (i.e., repetition) or by the other rule (i.e., switch). Otherwise switch costs reflect both the cost in performance when the task rule is switched versus repeated and a performance difference because trials are compared in which different tasks have to be performed.
}

context of negative images from the mean RT on trials involving a switch from the affective rule towards the nonaffective rule in the presence of negative images. Larger switch costs thus reflect poorer flexibility.

Heart rate variability Beat-to-beat heart rate was recorded continuously throughout the baseline phase $(10 \mathrm{~min})$ and the rest of the experiment using a telemetric heartbeat monitor (Polar V800; Polar Electro Oy, Kempele, Finland), which wirelessly received data from the chest strap worn by participants. Heart rate and interbeat interval sequences (i.e., RR intervals) were extracted and further analyzed with Kubios HRV Standard 3.1.0 software (Biosignal Analysis and Medical Imaging Group, n.d.; Tarvainen, Niskanen, Lipponen, Ranta-aho, \& Karjalainen, 2014). RR interval series were visually inspected for artefacts and corrected using Kubios artefact correction, which replaces detected artefact beats using cubic spline interpolation. We used time-domain-based RMSSD as our measure of vagally mediated HRV. To test the hypothesis of the current study, we used RMSSD based on the 10-min baseline phase, corresponding to resting HRV. Resting RMSSD $(M=42.53, S D=21.13)$ and resting HF-HRV $\left(\mathrm{ms}^{2}, 0.15\right.$ $0.40 \mathrm{~Hz})(M=1048, S D=1094)$ were highly correlated (Spearman $r=0.95, p<.001$ ); thus, only RMSSD was used to assess the associations with affective flexibility. The RMSSD reflects vagally mediated HRV and is relatively free of respiratory influences, as compared with high frequency parameters (Laborde et al., 2017). 


\section{Procedure}

Informed consent was obtained at the start of the session. After checking the exclusion criteria, the telemetric heartbeat monitor was put on, and beat-to-beat heart rate registration continued throughout the rest of the experiment. ${ }^{3}$ Participants then completed the self-report questionnaires (STAI-T and BDI), followed by the affective flexibility switching task. After the computer task, we measured the 10-min heart rate baseline period, in which participants were asked to sit quietly and to relax. At the end of this baseline period, we measured mood state with the VAS scales. Upon completion of the experiment, participants were debriefed about the nature of the study.

\section{Results}

We used R (R Core Team, 2019) for all analyses. Specifically, we used the R packages tidyverse (Wickham, 2017), psych (Revelle, 2018), and pastecs (Grosjean \& Ibanez, 2018) for data manipulation, exploration, and descriptive statistics. We used the package Hmisc (Harrell Jr, 2019) to calculate correlations. For the linear regression analysis, we used the packages stats (R Core Team, 2019), lmSupport (Curtin, 2018), and QuantPsyc (Fletcher, 2012). Finally, we used RMarkdown (Allaire et al., 2019), papaja (Aust \& Barth, 2018), knitr (Xie, 2019), qwraps2 (DeWitt, 2019), and kableExtra (Zhu, 2019) for the reporting and creation of the manuscript.

\section{Preliminary analysis}

Control variables Variables such as age, gender, average heart rate per minute (BPM), levels of anxiety, depression, and mood have been shown to affect resting HRV, and we therefore may want to control for these variables in further analyses. We first calculated Spearman correlations (some variables were not normally distributed) to test whether these variables were indeed related to resting HRV in our sample (see Table 1). Based on these correlations, we decided to control for BPM in further analyses. An independent $t$ test to check whether gender influenced HRV showed no significant effect, $t(42.48)=-0.21, p=.833$.

Affective flexibility data preparation Practice trials and the first trial of each test block in the affective flexibility task were excluded from analysis. In line with previous studies, we also included only trials that were preceded by a correct trial (Demanet, Liefooghe, \& Verbruggen, 2011; Mocan,

\footnotetext{
3 This study was part of a larger study in which we also measured trait resilience and emotion regulation with self-report questionnaires, and induced stress using a procedure based on the Trier Social Stress Test to measure stress reactivity. However, these measures will not be used to test the hypotheses of the current study.
}

Stanciu, \& Visu-Petra, 2014); because of post-error slowing and if the preceding trial is incorrect, it is ambiguous whether the current trial is a repetition or switch from the participant's perspective. To calculate accuracy, we thus divided the number of correct trials that were preceded by a correct response by the total number of trials (correct and incorrect) that were preceded by a correct response. One participant was excluded from further analysis because the percentage of errors was more than three standard deviations from the sample mean. Only correct trials that were also preceded by a correct response were included in the calculation of mean RT; this resulted in an average deletion of $11.29 \%$ of trials for each participant. To reduce the influence of outlying RTs, we replaced RT values 2.5 standard deviations above and below the mean RT within each participant and specific trial type by these upper and lower cutoff values, in line with previous studies (Genet et al., 2013; Greenwald, Nosek, \& Banaji, 2003; Malooly et al., 2013).

We used paired $t$ tests on RT on all repetition and switch trials, to confirm the presence of switch costs. RTs on repetition trials $(M=1,256 ; S D=251.57)$ were significantly lower than RTs on switch trials $(M=1,389 ; S D=278.33), t(108)=$ $-17.59, p<.001$. We then calculated nonaffective negative switch costs, affective negative switch costs, nonaffective positive switch costs, and affective positive switch costs.

\section{Descriptive statistics}

Mean and standard deviations for measures of trait anxiety, depressive symptoms, self-report measures of emotion and arousal, resting heart rate, resting heart rate variability, and switch costs from the affective flexibility task are shown in Table 2.

\section{Link between HRV and affective flexibility}

We performed a hierarchical linear regression analysis with resting RMSSD as the dependent variable. In a first step, we entered resting heart rate (BPM) to the model to control for the average heart rate per minute. In the second step, we entered the four types of switch costs: nonaffective negative, affective negative, nonaffective positive, and affective positive switch costs.

The model with just BPM was significant, $F(1,106)=$ $53.34, p<.001$, adj. $R^{2}=0.33$. Adding the four switch costs to the model near significantly improved fit, $F(4,102)=2.35$, $p=.059, \Delta R^{2}=0.06 .{ }^{4} \mathrm{BPM}$ was negatively related to

\footnotetext{
${ }^{4}$ Results are reported after exclusion of one outlier based on standardized residuals. With this case, adding switch costs to the model near significantly improved fit, $F(4,103)=2.16, p=.078, \Delta R^{2}=0.05$. The effect of nonaffective negative switch costs was similar, $\beta=0.19, t=2.30, p=.023$. When only adding nonaffective negative switch costs to the model with BMP, there is a significant improvement of fit, $F(1,105)=5.98, p=.016, \Delta R^{2}=$ 0.04 . The effect of nonaffective negative switch costs was similar, $\beta=0.19$. Running the analysis with HF-HRV, after transformation to its natural logarithm, resulted in similar findings.
} 
Table 1. Correlations

\begin{tabular}{|c|c|c|c|c|c|c|c|c|c|}
\hline & HRV & BPM & Age & STAI & BDI & Happiness & Sadness & Arousal & Anger \\
\hline \multicolumn{10}{|l|}{ HRV } \\
\hline BPM & $-0.56 * * *$ & & & & & & & & \\
\hline Age & -0.14 & $-0.25 * *$ & & & & & & & \\
\hline STAI & -0.03 & -0.02 & -0.06 & & & & & & \\
\hline BDI & 0.06 & -0.12 & -0.18 & $0.77 * * *$ & & & & & \\
\hline Happiness & -0.11 & 0.02 & 0.01 & $-0.23^{*}$ & $-0.22^{*}$ & & & & \\
\hline Sadness & -0.04 & -0.08 & -0.02 & $0.38 * * *$ & $0.32 * * *$ & 0.15 & & & \\
\hline Arousal & -0.01 & 0.03 & -0.10 & $0.22 *$ & 0.15 & $0.23 *$ & $0.50 * * *$ & & \\
\hline Anger & -0.14 & -0.04 & 0.04 & $0.24 *$ & 0.15 & 0.08 & $0.69 * * *$ & $0.44 * * *$ & \\
\hline Tension & 0.01 & 0.03 & -0.08 & $0.32 * * *$ & $0.28 * *$ & $<0.01$ & $0.48 * * *$ & $0.59 * * *$ & $0.37 * * *$ \\
\hline
\end{tabular}

$* p<.05 . * * p .01 . * * * p<.001 . \mathrm{HRV}=\mathrm{RMSSD}$ heart rate variability index; $\mathrm{BPM}=$ average heart rate per minute; $\mathrm{STAI}=$ trait anxiety; $\mathrm{BDI}=$ depressive symptoms

RMSSD with higher BPM related to lower HRV, $\beta=-0.59, t$ $=-7.47, p<.001$. Nonaffective negative switch costs were positively related to $\mathrm{RMSSD}, \beta=0.18, t=2.20, p=.030$. Lower nonaffective negative switch costs - that is, more efficient switching towards nonaffective aspects of negative information was thus related to lower RMSSD. Neither affective negative $(\beta<0.001)$, nonaffective positive $(\beta=0.12)$, nor affective positive switch costs $(\beta=0.09)$ were significant predictors of RMSSD (all $p \mathrm{~s}>.10$ ). See Fig. 2 for the partial correlation between RMSSD and nonaffective negative switch costs, controlling for BPM and other switch costs (negative affective switch costs, positive nonaffective switch costs, positive affective switch costs). Additional analyses with switch costs calculated as a percentage change from the nonswitch baseline are reported in the supplemental material.

Table 2. Descriptive statistics

\begin{tabular}{ll}
\hline & Data $(N=109)$ \\
\hline Trait anxiety (STAI) & $38.30(8.43)$ \\
Depressive symptoms (BDI) & $7.38(7.47)$ \\
Happiness & $49.00(26.00)$ \\
Sadness & $7.65(10.63)$ \\
Arousal & $17.96(19.08)$ \\
Anger & $2.81(5.12)$ \\
Tension & $13.52(15.94)$ \\
Resting HR & $75.17(8.94)$ \\
Resting RMSSD & $42.53(21.13)$ \\
Affective negative switch costs (ms) & $189.38(203.72)$ \\
Nonaffective negative switch costs (ms) & $77.17(203.93)$ \\
Affective positive switch costs (ms) & $119.02(165.98)$ \\
Nonaffective positive switch costs (ms) & $184.61(165.74)$ \\
\hline
\end{tabular}

Note. Mean (standard deviation); STAI = State-Trait Anxiety Inventory; $\mathrm{BDI}=$ Beck Depression Inventory

\section{Discussion}

The neurovisceral integration model summarizes the relationship between the central nervous system and the autonomous nervous system (Thayer et al., 2009; Thayer \& Lane, 2000). Vagally mediated heart rate variability provides an index of the influence of the parasympathetic nervous system - that is, a measure of cardiac vagal tone. The neurovisceral integration model aims to account for the complex interplay between autonomic, cognitive, and emotion regulation systems through their support by a common cortico-subcortical neural network. According to the model, higher resting HRV reflects better functioning of this neural network, supporting better

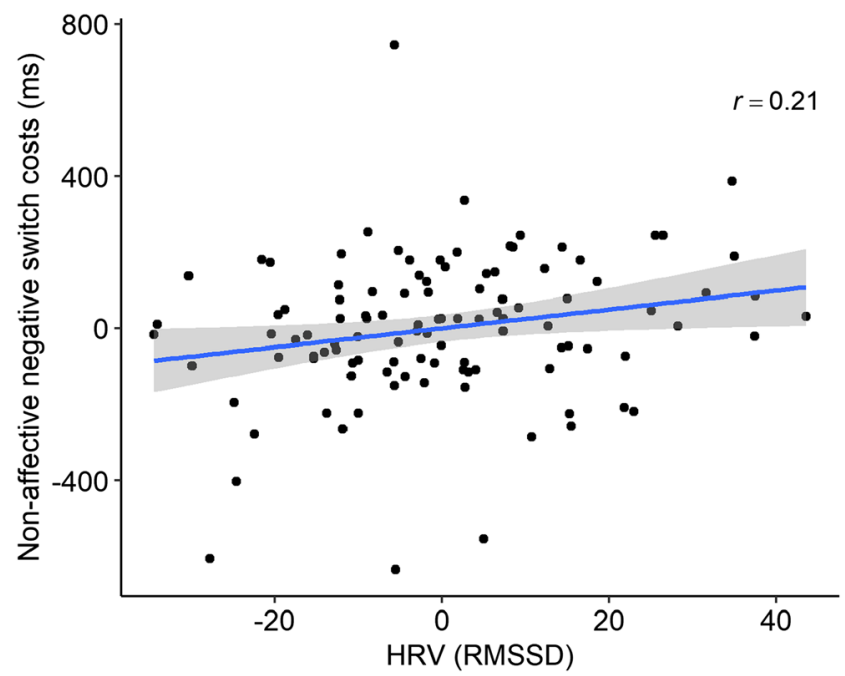

Fig. 2 Partial correlation between nonaffective negative switch costs and HRV (RMSSD). Residuals from regressing nonaffective negative switch costs on BPM, negative affective switch costs, positive nonaffective switch costs, and positive affective switch costs are plotted against the residuals from regressing RMSSD on BPM and the other switch costs. The Pearson correlation between these residual variables and the $95 \% \mathrm{CI}$ interval are displayed 
self-regulation and adaptation to changing situational demands. The relationship between autonomic regulation and cognitive control has previously been shown, with higher levels of resting HRV being associated with better monitoring and updating of working memory, attention control, response inhibition, and greater cognitive flexibility (Colzato et al., 2018; Hovland et al., 2012; Thayer et al., 2009; Zahn et al., 2016). Although greater cognitive flexibility is generally considered beneficial for adaptability to the environment, adding affective information can create 'contexts' where different cognitive responses could be required to meet the demands of a situation. The aim of the current study was therefore to investigate the association between vagally mediated resting HRV and cognitive flexibility in the context of affective information, to increase our understanding of the relationship between autonomic, cognitive, and affective systems.

More efficient shifting (i.e., greater flexibility) from nonaffective aspects towards affective aspects of positive information has previously been associated with more effective reappraisal use and smaller increases in anxiety over time (Malooly et al., 2013; Twivy et al., 2019). Because individual differences in anxiety (Friedman, 2007) and emotion regulation (Appelhans \& Luecken, 2006; Thayer \& Brosschot, 2005) have been linked to resting HRV, we expected more efficient shifting towards affective aspects of positive information also to be associated with higher resting HRV. However, we found no support for such a relationship. Whereas the majority of work on HRV and attention regulation for emotional information has focused on negative/threatening stimuli, it is possible that the link between resting HRV and attention regulation for positive information is relatively weaker.

We did find support for an association between more efficient shifting of attention from affective to nonaffective aspects of negative information and lower resting HRV. At first, this may seem to contradict the neurovisceral integration model that predicts higher resting HRV to be associated with more flexibility (Thayer et al., 2009; Thayer \& Lane, 2000). Although facilitated attention to negative information, lower resting HRV, and anxiety are indeed interlinked (Cisler \& Koster, 2010; Friedman, 2007; Park \& Thayer, 2014), research shows that anxious individuals actually show attentional avoidance when presented with negative information for longer durations. Such attentional avoidance may reflect a more strategic process underlying emotion regulation goals (Cisler \& Koster, 2010). In line with the avoidance model of anxiety and worry (Borkovec et al., 2004) and empirical evidence of attentional avoidance in anxiety, we recently found that more efficient shifting of attention from affective to nonaffective aspects of negative information predicted greater increases in anxiety across time (Twivy et al., 2019). As stimulus presentation in the task-switching paradigm is relatively long and we look at set shifting across trials, we are more likely tapping into such strategic processes. Our findings thus fit with the neurovisceral integration model, such that more efficient shifting of attention towards nonaffective aspects of negative information, which can facilitate attentional avoidance of negative information linked to anxiety (Cisler \& Koster, 2010), is related to lower resting HRV.

These findings also add to a growing notion that flexibility can be less adaptive in certain circumstances and that it is important to consider the (emotional) context (Parsons, Kruijt, \& Fox, 2016). When increased flexibility could facilitate attentional avoidance of negative information, it may obstruct learning that negative information does not always result in an aversive outcome, preventing threat associations to be altered by extinction learning or habituation (Borkovec et al., 2004). A relative inflexibility when shifting attention away from affective aspects of negative information could thus contribute to better functioning in the long term. Similarly, inflexibility when shifting attention away from affective aspects of negative information may also facilitate some form of 'situation awareness' - that is, comprehending the meaning of a situation. To make adequate decisions in (negative) situations, it is necessary to have situation awareness. Situation awareness has mostly been studied in contexts where human errors can occur (e.g., police command, health care). A previous study on situation awareness training in a policing context showed positive correlations between resting HRV and situation awareness scores (Saus et al., 2006). In an analogous way, adaptation to demands of a dynamic environment may rely on the regulation of attention in ways that allow for assessment of an affectively loaded situation to comprehend the meaning of the situation.

The association between less flexible shifting of attention from affective to nonaffective aspects of negative information and higher resting HRV is thus in agreement with the neurovisceral integration model such that it could facilitate self-regulation and adaptive functioning in a dynamic environment over time. Whereas previous work relates greater cognitive flexibility to higher resting HRV when measured as a single construct in the context of nonaffective information, we reveal the need for a more nuanced view when assessing attention regulation in the context of affective information, specifically concerning shifting to nonaffective aspects of negative information. Finally, it is important to note that the association between flexibility in shifting attention towards nonaffective aspects of negative information and HRV was small, but this is in line with meta-analytical evidence for the relationship between HRV and executive function (Zahn et al., 2016).

A few limitations to the study should be discussed. First, our sample consisted mostly of young, healthy adults, and so it remains unclear to what extent our findings generalize to other populations. Moreover, the nature of our population could also explain why no relationship was observed between trait anxiety, depressive symptoms, and HRV. Although previous research has found this relationship (Friedman, 2007; Kemp et al., 2010), our population reported 
low levels and relatively little variability in measures of trait anxiety (STAI-T) and depressive symptoms (BDI). Second, vagally mediated HRV seems to be sensitive to a range of factors such as age, gender, smoking, weight, cardioactive medication, physical activity, heart rate, and more (for an overview, see Laborde et al., 2017). Although we examined the relationship between some factors and HRV in the current sample, and excluded individuals using psychoactive or cardioactive medication, we cannot rule out that other unmeasured factors have influenced our results. Similarly, there is continued debate around respiratory control (Laborde et al., 2017). We did not measure respiratory rate and let participants breathe spontaneously, but we used RMSSD as our measure of vagally mediated HRV as this measure is less affected by breathing than HF-HRV, and the effects of respiration on parasympathetic indices of resting HRV have shown to be minimal (Laborde et al., 2017). Finally, it is important to note that correlation is not causation, so based on our findings we cannot draw any conclusions about the direction of the relationship between resting HRV and affective flexibility. As has been suggested before (Colzato et al., 2018), it would be interesting for future research to manipulate vagal control through transcutaneous vagal nerve stimulation and examine the effects on affective flexibility.

In summary, the current findings support the neurovisceral integration model (Thayer et al., 2009; Thayer \& Lane, 2000), such that individual differences in resting HRV relate to performance on a cognitive control task, possibly because of common underlying cortico-subcortical neural circuits supporting these processes. We extend previous work by investigating the association between resting HRV and cognitive flexibility (i.e., set shifting) in the context of affective information, demonstrating the need for a nuanced view on the complex interplay between vagally mediated HRV, cognitive control, and emotion.

Acknowledgements This research was supported by Grant FWO17/ PDO/201 for a postdoctoral fellowship of the Research FoundationFlanders awarded to Maud Grol. Rudi De Raedt is supported by Grant BOF16/GOA/017 for a Concerted Research Action of Ghent University.

Open practices statement The data and analysis scripts for this study are available on the Open Science Framework (https://osf.io/uqmfd/). This study was not preregistered.

\section{References}

Allaire, J., Xie, Y., McPherson, J., Luraschi, J., Ushey, K., Atkins, A., ... Iannone, R. (2019). Rmarkdown: Dynamic documents for R ( R Package Version 1.15) [Computer software]. Retrieved from https://github.com/rstudio/rmarkdown

Ansari, T. L., Derakshan, N., \& Richards, A. (2008). Effects of anxiety on task switching: Evidence from the mixed antisaccade task. Cognitive, Affective, \& Behavioral Neuroscience, 8(3), 229-238. doi:https://doi.org/10.3758/CABN.8.3.229

Appelhans, B. M., \& Luecken, L. J. (2006). Heart rate variability as an index of regulated emotional responding. Review of General
Psychology, 10(3), 229-240. doi:https://doi.org/10.1037/10892680.10.3.229

Aust, F., \& Barth, M. (2018). Papaja: Prepare reproducible APA journal articles with R Markdown (R Package Version 0.1.0.9842) [Computer software]. Retrieved from https://github.com/crsh/papaja

Balzarotti, S., Biassoni, F., Colombo, B., \& Ciceri, M. R. (2017). Cardiac vagal control as a marker of emotion regulation in healthy adults: A review. Biological Psychology, 130, 54-66. doi:https://doi.org/10. 1016/j.biopsycho.2017.10.008

Beck, A., Steer, R., \& Brown, G. (1996). Manual for the Beck Depression Inventory-II. San Antonio, TX: The Psychological Corporation.

Benarroch, E. E. (1993). The central autonomic network: Functional organization, dysfunction, and perspective. Mayo Clinic Proceedings, 68(10), 988-1001. doi:https://doi.org/10.1016/S0025-6196(12) $62272-1$

Biosignal Analysis and Medical Imaging Group. (n.d.). Kubios HRV Standard 3.1.0 [Computer software]. Finland: University of Kuopio.

Borkovec, T., Alcaine, O., \& Behar, E. (2004). Avoidance theory of worry and generalized anxiety disorder. In C. L. Turk \& D. S. Mennin (Eds.), Generalized anxiety disorder: Advances in research and practice (pp. 77-108). New York, NY: Guilford Press.

Brodal, P. (2010). The central nervous system-Structure and function. New York, NY: Oxford University Press.

Cisler, J. M., \& Koster, E. H. W. (2010). Mechanisms of attentional biases towards threat in anxiety disorders: An integrative review. Clinical Psychology Review, 30(2), 203-216. doi:https://doi.org/ 10.1016/j.cpr.2009.11.003

Colzato, L. S., Jongkees, B. J., de Wit, M., van der Molen, M. J. W., \& Steenbergen, L. (2018). Variable heart rate and a flexible mind: Higher resting-state heart rate variability predicts better taskswitching. Cognitive, Affective, \& Behavioral Neuroscience, 18(4), 730-738. doi:https://doi.org/10.3758/s13415-018-0600-x

R Core Team. (2019). R: A language and environment for statistical computing [Computer software]. Vienna, Austria: R Foundation for Statistical Computing. Retrieved from https://www.R-project. org/

Curtin, J. (2018). lmSupport: Support for linear models (R Package Version 2.9.13) [Computer software]. Retrieved from https:// CRAN.R-project.org/package=lmSupport

Davidson, R. J., Pizzagalli, D., Nitschke, J. B., \& Putnam, K. (2002). Depression: Perspectives from affective neuroscience. Annual Review of Psychology, 53, 545-574. doi:https://doi.org/10.1146/ annurev.psych.53.100901.135148

Demanet, J., Liefooghe, B., \& Verbruggen, F. (2011). Valence, arousal, and cognitive control: A voluntary task-switching study. Frontiers in Psychology, 2. doi:https://doi.org/10.3389/fpsyg.2011.00336

DeWitt, P. (2019). Qwraps2: Quick Wraps 2 (R Package Version 0.4.1) [Computer software]. Retrieved from https://CRAN.R-project.org/ package $=$ qwraps 2

Dishman, R. K., Nakamura, Y., Garcia, M. E., Thompson, R. W., Dunn, A. L., \& Blair, S. N. (2000). Heart rate variability, trait anxiety, and perceived stress among physically fit men and women. International Journal of Psychophysiology: Official Journal of the International Organization of Psychophysiology, 37(2), 121-133.

Fletcher, T. (2012). QuantPsyc: Quantitative Psychology Tools (R Package Version 1.5) [Computer software]. Retrieved from https:// CRAN.R-project.org/package=QuantPsyc

Friedman, B. H. (2007). An autonomic flexibility-neurovisceral integration model of anxiety and cardiac vagal tone. Biological Psychology, 74(2), 185-199. doi:https://doi.org/10.1016/j. biopsycho.2005.08.009

Gąsior, J. S., Sacha, J., Jeleń, P. J., Zieliński, J., \& Przybylski, J. (2016). Heart rate and respiratory rate influence on heart rate variability repeatability: Effects of the correction for the prevailing heart rate. Frontiers in Physiology, 7. doi:https://doi.org/10.3389/fphys.2016. 00356 
Genet, J. J., \& Siemer, M. (2011). Flexible control in processing affective and nonaffective material predicts individual differences in trait resilience Flexible control in processing affective and nonaffective material predicts individual differences in trait resilience, (February 2012), 37-41. doi:https://doi.org/10.1080/02699931. 2010.491647

Genet, J. J., Malooly, A. M., \& Siemer, M. (2013). Flexibility is not always adaptive: Affective flexibility and inflexibility predict rumination use in everyday life. Cognition \& Emotion, 27(4), 685-695. doi:https://doi.org/10.1080/02699931.2012.733351

Greenwald, A. G., Nosek, B. A., \& Banaji, M. R. (2003). Understanding and using the Implicit Association Test: I. An improved scoring algorithm. Journal of Personality and Social Psychology, 85(2), 197-216. doi:https://doi.org/10.1037/0022-3514.85.2.197

Grosjean, P., \& Ibanez, F. (2018). Pastecs: Package for analysis of spacetime ecological series (R Package Version 1.3.21) [Computer software]. Retrieved from https://cran.r-project.org/package=pastecs

Han, G., Helm, J., Iucha, C., Zahn-Waxler, C., Hastings, P. D., \& KlimesDougan, B. (2016). Are executive functioning deficits concurrently and predictively associated with depressive and anxiety symptoms in adolescents? Journal of Clinical Child and Adolescent Psychology, 45(1), 44-58. doi:https://doi.org/10.1080/15374416. 2015.1041592

Harrell Jr, F. E. (2019). Hmisc: Harrell miscellaneous (R Package Version 4.2-0) [Computer software]. Retrieved from https:// CRAN.R-project.org/package=Hmisc .

Hovland, A., Pallesen, S., Hammar, Å., Hansen, A. L., Thayer, J. F., Tarvainen, M. P., \& Nordhus, I. H. (2012). The relationships among heart rate variability, executive functions, and clinical variables in patients with panic disorder. International Journal of Psychophysiology: Official Journal of the International Organization of Psychophysiology, 86(3), 269-275. doi:https://doi. org/10.1016/j.ijpsycho.2012.10.004

Joormann, J., \& Stanton, C. H. (2016). Examining emotion regulation in depression: A review and future directions. Behaviour Research and Therapy, 86, 35-49. doi:https://doi.org/10.1016/j.brat.2016.07.007

Kemp, A. H., Quintana, D. S., Gray, M. A., Felmingham, K. L., Brown, K., \& Gatt, J. M. (2010). Impact of depression and antidepressant treatment on heart rate variability: A review and meta-analysis. Biological Psychiatry, 67(11), 1067-1074. doi:https://doi.org/10. 1016/j.biopsych.2009.12.012

Kim, K. L., Christensen, R. E., Ruggieri, A., Schettini, E., Freeman, J. B., Garcia, A. M., ... Dickstein, D. P. (2019). Cognitive performance of youth with primary generalized anxiety disorder versus primary obsessive-compulsive disorder. Depression and Anxiety, 36(2), 130-140. doi:https://doi.org/10.1002/da.22848

Laborde, S., Mosley, E., \& Thayer, J. F. (2017). Heart rate variability and cardiac vagal tone in psychophysiological researchRecommendations for Experiment planning, data analysis, and data reporting. Frontiers in Psychology, 8. doi:https://doi.org/10.3389/ fpsyg.2017.00213

Lang, P., Bradley, M., \& Cuthbert, B. (2008). International affective picture system (IAPS): Affective ratings of pictures and instruction manual (Technical Report A-8). Gainesville, FL: University of Florida.

Lee, J. K., \& Orsillo, S. M. (2014). Investigating cognitive flexibility as a potential mechanism of mindfulness in generalized anxiety disorder. Journal of Behavior Therapy and Experimental Psychiatry, 45(1), 208-216. doi:https://doi.org/10.1016/j.jbtep.2013.10.008

Lester, K. J., Lisk, S. C., Carr, E., Patrick, F., \& Eley, T. C. (2019). Associations between attentional bias and interpretation bias and change in school concerns and anxiety symptoms during the transition from primary to secondary school. Journal of Abnormal Child Psychology, 47(9), 1521-1532. doi:https://doi.org/10.1007/s10802019-00528-3
Malooly, A. M., Genet, J. J., \& Siemer, M. (2013). Individual differences in reappraisal effectiveness: The role of affective flexibility. Emotion, 13(2), 302-313. doi:https://doi.org/10.1037/a0029980

Miyake, A., Friedman, N. P., Emerson, M. J., Witzki, A. H., Howerter, A., \& Wager, T. D. (2000). The unity and diversity of executive functions and their contributions to complex "frontal lobe" tasks: A latent variable analysis. Cognitive Psychology, 41(1), 49-100. doi: https://doi.org/10.1006/cogp.1999.0734

Mocan, O., Stanciu, O., \& Visu-Petra, L. (2014). Relating individual differences in internalizing symptoms to emotional attention setshifting in children. Anxiety, Stress, and Coping, 27(5), 509-526. doi:https://doi.org/10.1080/10615806.2014.888419

Monfredi, O., Lyashkov, A. E., Johnsen, A.-B., Inada, S., Schneider, H., Wang, R., ... Boyett, M. R. (2014). Biophysical characterization of the underappreciated and important relationship between heart rate variability and heart rate. Hypertension (Dallas, Tex.: 1979), 64(6), 1334-1343. doi:https://doi.org/10.1161/HYPERTENSIONAHA. 114.03782

Niendam, T. A., Laird, A. R., Ray, K. L., Dean, Y. M., Glahn, D. C., \& Carter, C. S. (2012). Meta-analytic evidence for a superordinate cognitive control network subserving diverse executive functions. Cognitive, Affective, \& Behavioral Neuroscience, 12(2), 241-268. doi:https://doi.org/10.3758/s13415-011-0083-5

Ochsner, K. N., \& Gross, J. J. (2007). The neural architecture of emotion regulation. In J. J. Gross (Ed.), Handbook of emotion regulation (pp. 135-157). New York, NY: Guilford Press.

Park, G., \& Thayer, J. F. (2014). From the heart to the mind: Cardiac vagal tone modulates top-down and bottom-up visual perception and attention to emotional stimuli. Frontiers in Psychology, 5. doi: https://doi.org/10.3389/fpsyg.2014.00278

Parsons, S., Kruijt, A.-W., \& Fox, E. (2016). A cognitive model of psychological resilience. Journal of Experimental Psychopathology, 7(3), 296-310. doi:https://doi.org/10.5127/jep.053415

Pumprla, J., Howorka, K., Groves, D., Chester, M., \& Nolan, J. (2002). Functional assessment of heart rate variability: Physiological basis and practical applications. International Journal of Cardiology, 84(1), 1-14. doi:https://doi.org/10.1016/S0167-5273(02)00057-8

Revelle, W. (2018). Psych: Procedures for personality and psychological research [Computer software]. Evanston, IL: Northwestern University. Retrieved from https://www.scholars.northwestern.edu/ en/publications/psych-procedures-for-personality-andpsychological-research

Ruiz-Padial, E., Sollers, J. J., Vila, J., \& Thayer, J. F. (2003). The rhythm of the heart in the blink of an eye: Emotion-modulated startle magnitude covaries with heart rate variability. Psychophysiology, 40(2), 306-313. doi:https://doi.org/10.1111/1469-8986.00032

Saus, E.-R., Johnsen, B. H., Eid, J., Riisem, P. K., Andersen, R., \& Thayer, J. F. (2006). The effect of brief situational awareness training in a police shooting simulator: An experimental study. Military Psychology, 18(Suppl. 1), S3-S21. doi:https://doi.org/10.1207/ s15327876mp1803s_2

Spielberger, C., Gorsuch, R., Lushene, R., Vagg, P., \& Jacobs, G. (1983). Manual for the State-Trait Anxiety Inventory. Palo Alto, CA: Consulting Psychologists Press.

Sztajzel, J., Jung, M., \& Bayes de Luna, A. (2008). Reproducibility and gender-related differences of heart rate variability during all-day activity in young men and women. Annals of Noninvasive Electrocardiology: The Official Journal of the International Society for Holter and Noninvasive Electrocardiology, Inc, 13(3), 270-277. doi:https://doi.org/10.1111/j.1542-474X.2008.00231.x

Tarvainen, M. P., Niskanen, J.-P., Lipponen, J. A., Ranta-aho, P. O., \& Karjalainen, P. A. (2014). Kubios HRV-Heart rate variability analysis software. Computer Methods and Programs in Biomedicine, 113(1), 210-220. doi:https://doi.org/10.1016/j.cmpb.2013.07.024

Thayer, J. F., \& Brosschot, J. F. (2005). Psychosomatics and psychopathology: Looking up and down from the brain. 
Psychoneuroendocrinology, 30(10), 1050-1058. doi:https://doi.org/ 10.1016/j.psyneuen.2005.04.014

Thayer, J. F., \& Lane, R. D. (2000). A model of neurovisceral integration in emotion regulation and dysregulation. Journal of Affective Disorders, 61(3), 201-216.

Thayer, J. F., Friedman, B. H., \& Borkovec, T. D. (1996). Autonomic characteristics of generalized anxiety disorder and worry. Biological Psychiatry, 39(4), 255-266. doi:https://doi.org/10.1016/00063223(95)00136-0

Thayer, J. F., Hansen, A. L., Saus-Rose, E., \& Johnsen, B. H. (2009). Heart rate variability, prefrontal neural function, and cognitive performance: The neurovisceral integration perspective on self-regulation, adaptation, and health. Annals of Behavioral Medicine: A Publication of the Society of Behavioral Medicine, 37(2), 141153. doi:https://doi.org/10.1007/s12160-009-9101-z

Thayer, J. F., Åhs, F., Fredrikson, M., Sollers, J. J., \& Wager, T. D. (2012). A meta-analysis of heart rate variability and neuroimaging studies: Implications for heart rate variability as a marker of stress and health. Neuroscience \& Biobehavioral Reviews, 36(2), 747756. doi:https://doi.org/10.1016/j.neubiorev.2011.11.009

Twivy, E., Grol, M., \& Fox, E. (2019). Individual differences in affective flexibility predict change in anxiety and worry over time. Unpublished manuscript.

Umetani, K., Singer, D. H., McCraty, R., \& Atkinson, M. (1998). Twenty-four hour time domain heart rate variability and heart rate: Relations to age and gender over nine decades. Journal of the American College of Cardiology, 31(3), 593-601. doi:https://doi. org/10.1016/S0735-1097(97)00554-8
Van der Does, A. (2002). De Nederlandse versie van de Beck Depression Inventory-Tweede Editie [Beck Depression Inventory-Second Edition, Dutch Version]. Lisse, Netherlands: Swets \& Zeitlinger b.v.

Van der Ploeg, H., Defares, P., \& Spielberger, C. (2000). Handleiding bij de Zelf-Beoordelings Vragenlijst. Een Nederlandstalige bewerking van de Spielberger State-Trait Anxiety Inventory [Manual of the Self-Assessment Questionnaire: A Dutch version of the Spielberger State-Trait Anxiety Inventory]. Lisse, Netherlands: Swets; Zeitlinger b.v.

Wickham, H. (2017). Tidyverse: Easily install and load the 'tidyverse' (R Package Version 1.2.1) [Computer software]. Retrieved from https://CRAN.R-project.org/package=tidyverse

Williams, D. P., Feeling, N. R., Hill, L. K., Spangler, D. P., Koenig, J., \& Thayer, J. F. (2017). Resting heart rate variability, facets of rumination and trait anxiety: Implications for the perseverative cognition hypothesis. Frontiers in Human Neuroscience, 11. doi:https://doi. org/10.3389/fnhum.2017.00520

Xie, Y. (2019). Knitr: A general-purpose package for dynamic report generation in $\mathrm{R}$ (R Package Version 1.23) [Computer software]. Retrieved from https://rdrr.io/cran/knitr/

Zahn, D., Adams, J., Krohn, J., Wenzel, M., Mann, C. G., Gomille, L. K., ... Kubiak, T. (2016). Heart rate variability and self-control-A meta-analysis. Biological Psychology, 115, 9-26. doi:https://doi. org/10.1016/j.biopsycho.2015.12.007

Zhu, H. (2019). kableExtra: Construct complex table with 'kable' and pipe syntax (R Package Version 1.1.0) [Computer software]. Retrieved from https://CRAN.R-project.org/package=kableExtra

Publisher's note Springer Nature remains neutral with regard to jurisdictional claims in published maps and institutional affiliations. 